\title{
Territoires et Transports en Commun en Site Propre : réalisations, limites et perspectives
}

\section{Raymond Woessner et Marie-Agnès Lanneaux}

\section{OpenEdition}

Édition électronique

URL : http://journals.openedition.org/rge/3496

DOI : $10.4000 /$ rge.3496

ISSN : 2108-6478

Éditeur

Association des géographes de l'Est

Édition imprimée

Date de publication : 15 juin 2012

ISSN : 0035-3213

Référence électronique

Raymond Woessner et Marie-Agnès Lanneaux, «Territoires et Transports en Commun en Site Propre : réalisations, limites et perspectives », Revue Géographique de l'Est [En ligne], vol. 52 / 1-2 | 2012, mis en ligne le 04 février 2013, consulté le 08 septembre 2020. URL : http://journals.openedition.org/rge/ 3496 ; DOI : https://doi.org/10.4000/rge.3496

Ce document a été généré automatiquement le 8 septembre 2020

Tous droits réservés 


\title{
Territoires et Transports en Commun en Site Propre : réalisations, limites et perspectives
}

\author{
Raymond Woessner et Marie-Agnès Lanneaux
}

1 Les 15 et 16 septembre 2011, sous l'égide de la Commission de Géographie des Transports présidée par Jean Varlet, le colloque Territoires et Transports en Commun en Site Propre (TCSP) s'est tenu au sein de l'Université de Strasbourg. Outre les communications, il a permis deux visites de terrain, la première dans le réseau des transports en commun strasbourgeois et la seconde avec le tram-train entre Mulhouse et Thann. Le colloque a compté 17 communicants algériens, belges, espagnols et français ${ }^{1}$.

2 La problématique des TCSP génère des enjeux multiples lorsqu'il s'agit de reconstruire la ville sur elle-même. Dans l'histoire de l'aménagement du territoire de la France, que retenir des décennies bordières de l'an 2000 ? Une période de transition où l'État aura été à la peine pour mener à terme les grands projets d'infrastructures? Au fil des réformes, le dispositif réglementaire a été revu en profondeur, notamment en essayant d'entrer dans la logique de la subsidiarité typique de la construction européenne ainsi que dans celle de la durabilité grâce à l'élan donné par la conférence de Rio de Janeiro. Mais deux freins notables sont apparus : la complexité institutionnelle qui en a résulté et la faiblesse des moyens financiers octroyés.

3 Pourtant, l'urgence à refaire « la ville sans fin » selon l'expression de Françoise Choay existe. Lors de la publication du rapport Attali qui proposait de construire de nombreuses villes nouvelles, les praticiens se sont récriés, arguant qu'il vaut mieux repenser l'existant. Or les agglomérations ne cessent de se dilater, de se diluer et de dépendre de plus en plus des mobilités fondées sur le mode routier. Ainsi, le divorce est spectaculaire entre les désirs des ménages et des entreprises à la recherche d'un foncier abordable, et la volonté de la puissance publique, du haut en bas du millefeuille administratif, pour rendre la ville compacte et pour fonder les mobilités sur les transports publics (éventuellement aux mains d'entreprises privées). Lors de son 
passage à Strasbourg le 17 septembre 2009, Michel Mercier, ministre de l'espace rural et de l'aménagement du territoire, avait bien précisé qu'il «faut diminuer les transports» [routiers] parce que «le développement urbain en tâche d'huile, c'est terminé ».

Dès lors, les Transports en Commun en Site propre (TCSP) apparaissent comme l'élément structurant des agglomérations et des couronnes périurbaines en vue d'une durabilité. Le renoncement à la voiture passe par un phénomène push-pull. Le coût croissant de l'énergie et la congestion des réseaux routiers devraient détourner les usagers du mode routier dans le cas où l'offre TCSP existe. En effet, l'ambition des TCSP est d'offrir davantage de vitesse, de confort, de fréquences, d'intermodalité, de sécurité, d'accessibilité et de connexité, le tout avec un coût abordable pour les personnes puisque largement subventionné. Il s'agit donc de substituer en totalité ou en partie une organisation fondée sur l'illusion de l'initiative individuelle - je prends ma voiture et je vais où je veux quand je veux - par un système complexe de TCSP, avec de véritables défis pour les jeux d'acteurs concernés puisque la ville est à refaire en fonction des mobilités durables. Il faut attribuer de l'espace à des sites propres, apaiser et partager les couloirs de circulation, densifier l'habitat et les activités. Mais de nombreux points divers posent problème aux aménageurs, aux Autorités Organisatrices de Transport (AOT) et finalement aux élus chargés de prendre des décisions.

5 Les enjeux sont clairs à court et à moyen terme. Mais dans le temps long, les évolutions technologiques et sociétales sont difficiles à prévoir bien que la DATAR mène des études prospectives (France 2040). Si l'automobile devient propre, pilotée par des systèmes embarqués, plutôt louée qu'affichée en tant que symbole de la réussite sociale de l'individu, alors elle n'a pas dit son dernier mot. En matière de logement, la Maison sur la cascade de Frank Lloyd Wright, encastrée dans la "nature» forestière et aquatique, loin de la ville et de son agitation, restera peut-être un idéal onirique à atteindre. Mais si l'habitat compact devient un objet de désir, si l'e-commerce provoque l'enfrichement les zones commerciales de périphérie, si l'emploi se fixe près des haltes et gares, la fuite en avant qui se fait jusque dans les profondeurs du périurbain se tarira-t-elle?

6 Le choix du lieu du colloque n'est pas anodin, l'Alsace dispose de solides références en matière de TCSP. Elle apparaît comme une région interface qui s'inspire de solutions techniques rhénanes tout en fonctionnant sur un mode réglementaire français, où par exemple le préfet délivre in fine les autorisations de tracé et de vitesse des tramways, chose inconcevable outre-Rhin! Le TER 200 circule entre Strasbourg et Bâle depuis 1992. La trajectoire urbaine de Strasbourg a bifurqué à partir de 1994, lorsque le tramway est revenu dans la ville; ce dernier a permis de refonder une politique tournée vers la durabilité, parfois contre l'opinion publique (échec du référendum municipal de 2011 sur la généralisation des zones 30). Inspirée par l'exemple de Karlsruhe, Mulhouse a inauguré son tram-train en décembre 2010. Strasbourg s'apprête à lancer son premier Bus à Haut Niveau de Service (BHNS). Aujourd'hui, face à la pénurie grandissante de la ressource financière, il s'agit peut-être de s'inspirer d'innovations frugales venues de villes émergentes, à l'image du Bus Rapid Transit (BRT) de Curitiba au Brésil. 
7 Le colloque de Strasbourg a fait apparaitre les TCSP comme le facteur structurant de l'aménagement des territoires urbains et périurbains. Grâce aux différentes contributions, ce voyage au pays des TCSP s'articule autour quatre problématiques.

- Le projet politique. Un réseau de TCSP redessine les contours de l'espace public. Un retour sur l'histoire montre les différences de comportement des pays d'Europe lors du démantèlement des réseaux historiques de tramway. Plusieurs intervenants ${ }^{2}$ ont pu démontrer que les élus s'emparaient d'un mode donné en fonction de la doxa du moment. La mode plutôt que le mode! Dans les années 1990-2000, le marketing territorial a favorisé le « retour triomphal » du tramway devenu un « objet-fétiche », voire « le jouet du maire ». En Espagne, il est question de « prestige global » grâce au tramway. En France, la fin d'un cycle est atteinte avec les derniers travaux en cours et le probable abandon de certains projets. Déjà, le BHNS prend le relais dans les stratégies de communication. Et l'entreprise privée est appelée à la rescousse pour assurer un service public. La nature des relations entre les élus, le secteur privé et les techniciens en général constituent un facteur explicatif de l'émergence et de la configuration des réseaux de TCSP.

- L'évaluation d'un TCSP. Une fois le TCSP sur ses rails ou sur ses pneus, qu'advient-il ? Jusqu'à quel point le projet est-il conforme aux attentes ? Comme le montrent un certain nombre de communications ${ }^{3}$, dans bien des cas, la fréquentation est supérieure aux prévisions. Les villes se disputent les techniciens talentueux. En reproduisant leur savoirfaire, ceux-ci créent une forme d'homogénéisation territoriale dans les différentes collectivités où ils travaillent. Mais il est bien difficile de produire une évaluation objective $\mathrm{du}$ système ainsi construit. Les auteurs des bilans sont souvent juges et parties; l'autocongratulation est souvent de rigueur. La morphologie même du réseau peut biaiser la comparaison avec la situation antérieure lorsqu'on s'intéresse aux dessertes, aux vitesses et aux temps de parcours. Les réseaux de personnes, fondés sur une articulation entre les élites locales et les centres de décision comme des lobbies venant de l'extérieur, expliquent la genèse des territoires TCSP ; mais, au cas par cas, les «stratégies de paravent » rendent ces stratégies opaques. C'est pourquoi la création d'observatoires indépendants, à l'image de ce que loi impose pour les autoroutes, serait un gage de connaissance objective.

- Les densités et la forme urbaine. Un réseau de TCSP relie les différents points de la ville, de la banlieue et jusqu'aux territoires périurbanisés. Joue-t-il un rôle curatif en recadrant a posteriori l'urbanisme ou bien a-t-il un rôle préventif en précédant la construction de nouveaux quartiers? Les comparaisons avec d'autres pays ${ }^{4}$ montrent à quel point l'histoire et la culture interfèrent implicitement avec cette problématique. Dans le monde rhénan, les densités de population, la granulométrie (ou si l'on veut le semis et la taille des agglomérations) ainsi que l'adhérence (l'urbanisation qui se colle à l'existant) ne fonctionnent pas comme en France. En outre, dans le détail des réseaux, les échelles sont multiples; les limites des différents périmètres réglementaires cisaillent fréquemment la continuité de l'action publique. Il faut trouver des solutions, âprement négociées. Un texte réglementaire national peut ainsi être retouché pour satisfaire à la résolution d'un problème local. Sur un parcours relativement long, comment faire un choix entre la vitesse et la finesse de la desserte? Comment trouver les solutions optimales en matière d'intermodalité ? Comment connecter les espaces délaissés à l'organisme urbain?

- L'irruption des pays émergents. Si les pays rhénans «en avance » indiquent une voie à suivre, quoique difficilement transposable en France, qu'en est-il des pays émergents ?5 Puissants dans les villes du centre de l'Europe, les TCSP débordent sur les périphéries. L'Espagne et le Portugal ont ainsi vigoureusement développé leurs réseaux en s'inspirant des techniques et des expériences réussies. A présent, les pays du Maghreb sont entrés dans 
le jeu. Leurs grandes villes disposent déjà ou vont inaugurer des réseaux TCSP, créant ainsi concrètement une certaine forme d'Euroméditerranée. Les pays émergents appliquent-ils les pratiques des européens par imitation? Ou bien leur créativité intrinsèque produit-elle de nouveaux modèles qui méritent - outre l'intérêt qu'ils présentent pour les mobilités in situ une réflexion pour les adapter sous nos latitudes?

8 Ainsi, les travaux du colloque apparaissent comme un manifeste pour la défense et illustration des TCSP sur la base d'une analyse critique des réalisations existantes ou à venir. En effet, la croissance - ou la décroissance - urbaine livrée à une planification défaillante produit un objet spatial de qualité et de fonctionnalité pour le moins médiocres. Avec les TCSP, le développement durable, le beau urbain et le "réenchantement de la ville» sont au cœur des préoccupations des intervenants du colloque. L'utopie s'est même invitée au débat lorsqu'il a été question du tram-fret (Mathieu Strale, non-publié ici). Va-t-on vers la ville aux caractéristiques homogènes partout dans le monde, tiraillée entre l'image naïve de la liberté individuelle associée à la puissance dévastatrice du marché et des communautarismes face à la volonté planificatrice avec comme objectif principal le développement durable?

\section{NOTES}

1. Ses organisateurs sont Estelle Baehrel, secrétariat LIVE, Université de Strasbourg, Joël Forthoffer, SNCF Strasbourg, Marie-Agnès Lanneaux, Lille 3 et Raymond Woessner. Le comité scientifique regroupe en outre Antoine Beyer, IFSTTAR, Laurent Chapelon, Montpellier 3, Anne Hecker, Nancy 2, Robert Marconis, professeur émérite, Colette Ranély Vergé-Depré, Université des Antilles et de la Guyane, Jean-François Troin, professeur retraité, Jean Varlet, Université de Savoie, Michel Vrac, Université de Franche-Comté, Jean-Pierre Wolff, Université de Toulouse - Le Mirail, et Pierre Zembri, Université de Cergy-Pontoise.

2. Jean-Pierre Wolff, Vincent Gagnière pour les villes françaises, Colette Ranély Vergé-Dépré pour l'agglomération foyalaise, Miguel Pazos Oton pour les villes espagnoles.

3. Pierre Zembri pour les villes françaises, Cyprien Richer / Sophie Hasiak pour le Nord de la France, Laurent Fouillé pour Rennes.

4. Belinda Redondo pour les villes françaises, Matthieu Drevelle pour Montpellier, Juliette Maulat pour Toulouse, Jean Varlet pour Lyon, Joël Forthoffer, Anne Hecker pour la France et l'Allemagne.

5. Guy Chemla et Clémence Montagne pour Ahmedabad. 


\section{AUTEURS}

\section{RAYMOND WOESSNER}

Professeur de géographie à l'Université Paris 4, Espaces, Nature et Culture UMR 8185. Lors de la préparation du colloque : Laboratoire Image, Ville et Environnement ERL 7230.

raymond.woessner[chez]wanadoo.fr

\section{MARIE-AGNÈS LANNEAUX}

Maître de conférences à l'Université de Lille 3. MRTE - Université Cergy-Pontoise. marieagnes.lanneaux[chez]univ-lille3.fr 